\title{
La génétique des Insectes vecteurs de maladies Programme de l'O.M.S.
}

\author{
par R. PAL \\ Organisation Mondiale de la Santé, Genève, Suisse
}

\begin{abstract}
Résumé
Depuis quelques années, l'O.M.S. attache une importance croissante aux recherches génétiques intéressant la lutte antivectorielle. Les travaux en cours peuvent être groupés sous les grandes rubriques suivantes: $a$ ) génétique de la résistance, $b$ ) systèmes génétiques présents chez les principales espèces de vecteurs, $c$ ) obtention de souches d'insectes ayant des caractéristiques génétiques bien définis, et $d$ ) recherche de méthodes de lutte fondées directement sur les mécanismes génétiques. Certaines de ces questions sont passées en revue dans l'article.
\end{abstract}

\section{Summary}

During the past few years WHO has placed an increasing emphasis on genetic research on vector control. The following are general categories of investigations being carried out: a) genetics of resistance, $b$ ) genetic systems operating in important vector species, c) the development of genetically defined strains of insects, and $d$ ) the search for control measures based directly upon genetic mechanisms. Some of these topics are discussed.

\section{Introduction.}

La résistance des insectes aux insecticides est depuis quelques années une menace des plus graves pour la lutte contre les infections transmises à l'homme par des vecteurs ou pour l'éradication de ces maladies. S'agissant, comme on l'a montré, d'un caractère héréditaire, elle pose un problèmə extrêmement complexe qui ne peut être résolu sans 
une connaissance préalable de la génétique des insectes vecteurs. Certains de ces derniers se prêtent d'ailleurs particulièrement bien aux études fondamentales relevant de nombreux domaines de la génétique.

En 1962, l'O.M.S. a entrepris un vaste programme de recherches sur la génétique des vecteurs. Au cours des sept dernières années, elle a organisé un certain nombre de réunions dont l'objet était de faire le bilan des connaissances acquises en la matière et d'indiquer les points exigeant de nouvelles recherches. Ces réunions sont les suivantes:

1) Groupe scientifique des recherches sur la génétique des vecteurs et de la résistance aux insecticides (1).

2) Groupe scientifique des souches normalisées d'insəctes intéressant la santé publique (2).

3) Groupe de coordination de la lutte génétique contre les insectes intéressant la santé publique (3).

4) Groupe scientifique de la cytogénétique des vecteurs de maladies humaines (4).

Les informations autorisées recueillies grâce à ces réunions ont permis de renseigner chercheurs et laboratoires collaborateurs sur les recherches les plus utiles à entreprendre.

\section{Génétique de la résistance.}

L'O.M.S. a patronné sur la résistance aux insecticides des travaux qui ont porté sur à peu près tous les aspects du problèmə et notamment l'aspect génétique. L'étude de la fréquence des gènes résistants dans les populations d'insectes, de leur localisation chromosomique, de leur mode de transmission et celle du spectre de résistance aux différents insecticides ont particulièrement été poussées. Ces études ont permis de déterminer le degré de développement que peut atteindre la résistance et de déceler les génotypes résistants bien avant que leur fréquence soit suffisante pour faire échec aux programmes de lutte antivectorielle.

\section{Génétique formelle et cytogénétique.}

L'O.M.S. coordonne actuellement un programme complet de recherches de génétique formelle et de cytogénétique concernant les vecteurs. Les cartes chromosomiques de la plupart des espèces américaines et européennes d'anophèles ont été achevées au cours des dernières années. L'existence chez Anopheles gambiae, d'un complexe d'espè-

(1) 1964, Génétique des vecteurs et résistance aux insecticides. Org. mond. Santé Sér. Rapp. techn., $n^{\circ} 268$.

(2) 1966, Standardized Strains of Insects of Public Health Importance. Bull. Org. mond. Santé 34, $437-460$. 421-438.

(3) 1968, Genetic Control of Insects of Public Health Importance. Bull. Org. mond. Santé, 38,

(4) 1968, Cytogénétique des vecteurs de maladies humaines. Org. mond. Santé Sér. Rapp. techn., $\mathrm{n}^{\circ} 398$. 
ces a été mise en évidence et les recherches effectuées dans les laboratoires collaborateurs de l'O.M.S. ont montré que ces espèces - que l'on ne peut distinguer par leur morphologie - peuvent être identifiées par leurs chromosomes sexuels. La découverte récente d'espèces sœurs dans le genre Simulium a également encouragé les travaux entrepris pour dresser la carte chromosomique des simulies de diverses régions d'Afrique et du Canada.

Au cours des dernières années, de nouveaux renseignements ont été obtenus sur le caryotype de plusieurs espèces. Les cartes chromosomiques des glandes salivaires de diverses spèces d'anophèles sont actuellement disponibles. Des cartes de linkage concernant Musca domestica et Aedes aegypti ont été préparées. Enfin, les mécanismes de stérilité : incompatibilité cytoplasmique, translocation chromosomique, perturbation de la proportion des sexes, etc., ont été étudiés en vue de leur application à la lutte génétique contre les espèces vectrices.

\section{Souches types et souches à marqueurs.}

Sous l'égide de l'O.M.S., des élevages de souches de vecteurs de constitution génétique connue ont été établis. Ils concernent des souches de mouches domestiques (souches de référence pour les insecticides, souches porteuses de marqueurs chromosomiques), du complexe Culex (souches endogames de référence pour les insecticides, souches incompatibles et souches à marqueurs), ainsi que d'Aedes aegypti (souches à marqueurs) et de certains anophèles. Ces diverses souches sont fournies sur demande adressée aux Centres internationaux de référence de l'O.M.S. L'O.M.S. a, en particulier, créé des Centres internationaux de référence pour l'entretien et la distribution des souches types de Musca domestica (Pavie, Italie), du complexe Culex (Mayence, Allemagne) et d'Anophèles (Londres, Royaume-Uni).

\section{Lutte génétique contre les vecteurs.}

$\mathrm{Au}$ cours des quelques dernières années, les recherches concernant la lutte contre les insectes par manipulation génétique, ont considérablement progressé. Les méthodes génétiques utilisées pour combattre ou exterminer une espèce sont sélectives, et leur application ne met en danger aucun des autres êtres vivants de la région considérée.

Parmi ces méthodes figure le lâcher de mâles stériles, obtenus par irradiation ou par traitement chimique. Les expériences récentes de Laven (1968 (3, 5) sur Culex fatigans, faites sous l'égide de l'O.M.S. dans la nature à Rangoon (Birmanie) ont apporté pour la première fois la preuve que l'incompatibilité naturelle pouvait être exploitée avec succès sans qu'on ait besoin de recourir à l'irradiation ou à la chimiostérilisation. Encouragée par ces résultats, l'Organisation projette un essai à grande échelle de lutte génétique contre les culicinés en Inde. Elle étudie également les possibilités qu'offre dans le cas d'Anophèles gambiae, principal vecteur du paludisme en

(5) Laven (H.), 1967. - Eradication of Culex pipiens fatigans through cytoplasmic incompatibility. Nature, 216, 383-384. 
Afrique, la lutte génétique basée sur l'utilisation d'un mécanisme différent, celui de la stérilité des hybrides. Un essai préliminaire mené par Davidson et al. (1969) (6) dans une petite ville proche de Bobo-Dioulasso (Haute-Volta) a mis en évidence l'utilité potentielle de ce mécanisme, mais certains problèmes exigent une étude plus poussée. L'Organisation examine, en outre, dans quelle mesure la méthode peut s'appliquer à Aedes aegypti, aux glossines, aux tiques et aux triatomes.

\section{Diffusion des renseignements.}

Avec l'extension des recherches sur la génétique des vecteurs, l'amélioration des communications entre chercheurs est devenue d'urgente nécessité. C'est la raison pour laquelle l'O.M.S. a organisé, sur le modèle du service d'information sur la drosophile et du service d'information sur Tribolium, un service d'information sur la génétique des vecteurs $(7,8)$. Il consiste à faire paraître, périodiquement, une liste type des espèces vectrices et des mutants, des notes techniques et scientifiques, une bibliographie des travaux publiés, des listes de rapports inédits et des demandes de matériel génétique. Il comporte également un répertoire alphabétique et géographique des personnes travaillant dans ce domaine.

Pour remédier à la pénurie mondiale de personnel qualifié en la matière, l'O.M.S. a organisé un cours de génétique des vecteurs. Le premier, donné en 1968, avec la collaboration de l'Université Notre-Dame à Indiana (Etats-Unis d'Amérique) a été suivi par 26 participants venant de 13 pays; durant les six semaines de discussions, tous les aspects du sujet ont été examinés. L'accent a notamment été mis sur les possibilités d'application de la génétique dans les programmes pratiques. L'O.M.S. a, en outre, attribué un certain nombre de bourses d'études en génétique des vecteurs.

L'absence de tout traité d'ensemble sur cette science nouvelle a incité l'O.M.S. à préparer un ouvrage de génétique des insectes vecteurs de maladies, publié en 1967 (9), qui rassemble les vues de 31 experts faisant autorité sur tous les aspects de la question.

(6) Davidson (G.), 1969. - A field attempt to assess the mating competitiveness of sterile males produced by crossing two member species of the Anopheles gambiae complex. WHO/VBC/69.149 et $\mathrm{WHO} / \mathrm{Mal} / 69.687$.

(7) 1966, Service d'information sur la génétique des vecteurs, $\mathrm{n}^{\circ} 1, \mathrm{VBC} / 67.1$.

(8) 1968, Vector Genetics, Mutation and Stock List of Arthropod Vectors, WHO/VBC/68.76.

(9) Wright (J.-W) et Pal (R.), 1967. - Genetics of Insect Vectors of Disease, Elsevier Publishing Co., Amsterdam. 